\title{
Efficacy of the Product Spinosad an Insecticide Used in the Control of Tomato Leafminer (Tuta absoluta - Meyrick, 1917)
}

\author{
Elena BRATU ${ }^{\left.1^{*}\right)}$, Alexandra Maria PETCUCI ${ }^{2 *}$ and Gabriela SOVAREL ${ }^{1)}$ \\ 1) Plant Protection Laboratory, Research and Development Institute for Vegetable and Flower Growing, \\ Calea București no. 22, Vidra-077185, Ilfov, Romania; gabriela_sovarel@yahoo.com \\ 2) Biology Manager - Romania \& Adria, Dow AgroSciences Export SAS, Rep. Office Bucharest; \\ *) corresponding authors: bratu.bpe@icdlfvidra.ro; ampetcuci@dow.com
}

BulletinUASVM Horticulture 72(1) / 2015

Print ISSN 1843-5254, Electronic ISSN 1843-5394

DOI:10.15835/buasvmcn-hort:10876

\begin{abstract}
Product Spinosad was applied at doses varying between 48 to $120 \mathrm{~g}$ a.i./ha (one or 2 times at 14 days interval) against tomato leafminer, Tuta absoluta, in tomato (Prekos F1) grown in plastic tunnels. Observations concerning product phytotoxicity, \% of attacked area/leaf and plant, were made at one week after each treatment, and 4 weeks after first treatment. Efficacy was determined from the average of the two indicators monitored. Best results (99.8 \%) were obtained from spinosad at $120 \mathrm{~g}$ a.i./ha 28 days after a single application. Comparison standardcontrol emamectin-benzoate used once at a rate of $14.25 \mathrm{~g}$ a.i./ha had comparable efficacy $(99.0 \%)$ at the same evaluation, while a second standard product, imidacloprid at $200 \mathrm{~g}$ a.i./ha rate only reached $81.9 \%$ after 28 days. No phytotoxic effects were observed.
\end{abstract}

Keywords: efficacy, emamectin-benzoate, spinosad, Tuta absoluta

\section{INTRODUCTION}

Currently, tomato leafminer (Tuta absoluta) represents, one of the most economically important pests of tomato. Originally from South America (Peru, Columbia, etc.), tomato leafminer was first identified in Spain in 2006 and has spread rapidly within Europe (Desneux et al., 2010 ) and outside of the European continent. The species now occurs from Latvia in the north, Turkmenistan and Iran in the east, United Kingdom in the west and into Greece and Turkey in the south. In Romania, miner moth was first recognized in the North West zone (Satu Mare, 2009) and one year later was found near Bucharest, in greenhouse crops. Rapid expansion of resistant pest populations to a range of different pesticides make tomato leafminer, one of the most dangerous pest of commercial tomato crops (Antonio Cesar dos Santos et al., 2011).

Aims and objectives. Tuta absoluta has developed resistance to a range of insecticide products including some of the most widely used products such as the pyrethroids (e.g., gamma-cyhalothrin). We wanted to evaluate the product spinosad determine if it could be an effective solution for integrated control of this pest. Spinosad contains spinosyn $A$ and spinosyn $D$, which are two naturally occurring secondary metabolites of the soil actinomycete Saccharopolyspora spinosa. The research report here had as objectives to determine the efficacy and persistence of spinosad compared to standard products.

\section{MATERIALS AND METHODS}

The experiment was performed in a plastic tunnel, planted to tomato ("Prekos F1" cultivar) in the second cycle. A randomized complete block experimental design with controls included was used. The experiment evaluated three spinosad treatments: 2 applications of $48 \mathrm{~g}$ a.i./ha; 2 applications of $60 \mathrm{~g}$ a.i./ha; and one application of $120 \mathrm{~g}$ a.i./ ha. Two commercial standard products were included in the trial: emamectin-benzoate applied at $7.12 \mathrm{~g}$ a.i./ha 2 times and $14.25 \mathrm{~g}$ a.i./ha applied once; and imidacloprid applied at $100 \mathrm{~g}$ a.i/ha twice and $200 \mathrm{~g}$ a.i. l/ha applied once. The interval between applications was 14 days. Observations were made at 2 and 7 days after each treatment, and 14 days after the second treatment. Data were recorded on product phytotoxicity, percent of attacked area/leaf, and plant. Statistical analysis was performed.

\section{RESULTS AND DISCUSSION}

Knock down activity varied between $21 \%$ and 75 $\%$ in the case of attacked area/leaf (Table 1), and from 
Tab. 1. Dynamic Spinosad product effectiveness in reducing attack area/leaf of Tuta absoluta pest at tomato crop under plastic tunnel

\begin{tabular}{|c|c|c|c|c|c|c|c|}
\hline \multirow[b]{2}{*}{ Product } & \multirow{2}{*}{$\begin{array}{c}\text { Rate } \\
\text { (g a.i./ ha) }\end{array}$} & \multicolumn{6}{|c|}{ Efficacy (\%) after: } \\
\hline & & 2 DAA1 & 7 DAA1 & $\begin{array}{c}14 \text { DAA1- } \\
0 \text { DAA2 }\end{array}$ & $\begin{array}{c}16 \text { DAA1- } \\
2 \text { DAA2 }\end{array}$ & $\begin{array}{c}21 \text { DAA1- } \\
7 \text { DAA2 }\end{array}$ & $\begin{array}{c}28 \text { DAA1- } \\
14 \text { DAA2 }\end{array}$ \\
\hline Spinosad $240 \mathrm{SC}$ & $48.0(2 \mathrm{X})$ & 21.0 & 53.3 & 74.9 & 92.3 & 97.2 & 98.2 \\
\hline Spinosad $240 \mathrm{SC}$ & $60.0(2 \mathrm{X})$ & 61.1 & 74.8 & 82.5 & 97.5 & 99.7 & 99.3 \\
\hline Spinosad 240 SC & $120.0(1 \mathrm{X})$ & 75.0 & 75.0 & 99.7 & 100.0 & 99.9 & 99.8 \\
\hline Emamectin-benzoate $0.95 \mathrm{WG}$ & $7.12(2 X)$ & 50.0 & 75.0 & 94.3 & 97.7 & 94.2 & 98.2 \\
\hline Emamectin-benzoate $0.95 \mathrm{WG}$ & $14.25(1 \mathrm{X})$ & 52.8 & 75.0 & 98.9 & 98.5 & 96.2 & 99.3 \\
\hline Imidacloprid $200 \mathrm{SC}$ & $100.0(2 \mathrm{X})$ & 38.4 & 70.4 & 69.0 & 70.8 & 68.4 & 65.8 \\
\hline Imidacloprid $200 \mathrm{SC}$ & $200.0(1 \mathrm{X})$ & 49.6 & 74.6 & 94.2 & 95.9 & 79.4 & 81.0 \\
\hline Untreated check - attack level \% & - & 0.605 & 1.280 & 2.510 & 2.923 & 14.74 & 19.25 \\
\hline LSD $(\mathrm{P}=.05)-\%$ attack area/leaf & & 0.9097 & 0.7261 & 0.7888 & 1.0485 & 2.8638 & 3.6772 \\
\hline Standard deviation & & 0.6185 & 0.4937 & 0.5363 & 0.7129 & 1.9472 & 2.5002 \\
\hline
\end{tabular}

Tab. 2. Dynamic of Spinosad product effectiveness in reducing attack area/plant of Tuta absoluta pest at tomato crop under plastic tunnel

\begin{tabular}{|c|c|c|c|c|c|c|c|}
\hline \multirow[b]{2}{*}{ Product } & \multirow[b]{2}{*}{$\begin{array}{c}\text { Rate } \\
\text { (g a.i./ ha) }\end{array}$} & \multicolumn{6}{|c|}{ Efficacy (\%) after: } \\
\hline & & 2 DAA1 & 7 DAA1 & $\begin{array}{l}\text { 14 DAA1- } \\
0 \text { DAA2 }\end{array}$ & $\begin{array}{l}16 \text { DAA1- } \\
2 \text { DAA2 }\end{array}$ & $\begin{array}{l}21 \text { DAA1- } \\
7 \text { DAA2 }\end{array}$ & $\begin{array}{l}28 \text { DAA1- } \\
14 \text { DAA2 }\end{array}$ \\
\hline Spinosad 240 SC & $48.0(2 X)$ & 42.3 & 47.5 & 71.8 & 95.0 & 98.7 & 99.0 \\
\hline Spinosad 240 SC & $60.0(2 X)$ & 50.0 & 74.6 & 88.6 & 97.1 & 99.6 & 99.6 \\
\hline Spinosad 240 SC & $120.0(1 \mathrm{X})$ & 50.0 & 87.5 & 92.5 & 99.2 & 99.9 & 99.7 \\
\hline Emamectin-benzoate $0.95 \mathrm{WG}$ & $7.12(2 X)$ & 25.0 & 87.5 & 96.0 & 97.5 & 96.9 & 98.1 \\
\hline Emamectin-benzoate $0.95 \mathrm{WG}$ & $14.25(1 \mathrm{X})$ & 50.0 & 87.5 & 98.5 & 98.9 & 97.6 & 98.7 \\
\hline Imidacloprid $200 \mathrm{SC}$ & $100.0(2 X)$ & 25.0 & 67.8 & 57.8 & 82.2 & 72.5 & 71.7 \\
\hline Imidacloprid $200 \mathrm{SC}$ & $200.0(1 \mathrm{X})$ & 47.5 & 73.1 & 92.8 & 92.7 & 84.8 & 82.7 \\
\hline Untreated check - attack level \% & - & 0.418 & 0.740 & 3.688 & 4.168 & 22.125 & 25.050 \\
\hline LSD $(\mathrm{P}=.05)-\%$ attack area/ & & 0.7829 & 0.4119 & 0.9512 & 1.457 & 3.6904 & 4.1387 \\
\hline Standard deviation & & 0.5323 & 0.2801 & 0.6467 & 0.9906 & 2.5092 & 2.8139 \\
\hline
\end{tabular}

Tab. 3. Dynamic of Spinosad product effectiveness in reducing Tuta absoluta attack at tomato crop under plastic tunnel

\begin{tabular}{|c|c|c|c|c|c|c|c|}
\hline \multirow[b]{2}{*}{ Product } & \multirow[b]{2}{*}{$\begin{array}{c}\text { Rate } \\
\text { (g a.i./ ha) }\end{array}$} & \multicolumn{6}{|c|}{ Efficacy (\%) after: } \\
\hline & & 2 DAA1 & 7 DAA1 & 14 DAA1- & 16 DAA1- & 21 DAA1- & 28 DAA1- \\
\hline Spinosad 240 SC & $48.0(2 X)$ & 31.7 & 50.4 & 73.4 & 93.7 & 98.0 & 98.6 \\
\hline Spinosad 240 SC & $60.0(2 X)$ & 55.5 & 74.7 & 85.6 & 97.3 & 99.7 & 99.5 \\
\hline Spinosad 240 SC & $120.0(1 \mathrm{X})$ & 62.5 & 81.3 & 96.1 & 99.6 & 99.9 & 99.8 \\
\hline Emamectin-benzoate $0.95 \mathrm{WG}$ & $7.12(2 x)$ & 375 & 81.3 & 95.2 & 97.6 & 95.6 & 98.2 \\
\hline Emamectin-benzoate $0.95 \mathrm{WG}$ & $14.25(1 \mathrm{X})$ & 51.4 & 81.3 & 98.7 & 98.7 & 96.9 & 99.0 \\
\hline Imidacloprid 200 SC & $100.0(2 X)$ & 31.7 & 69.1 & 63,4 & 76.5 & 70.5 & 68.8 \\
\hline Imidacloprid 200 SC & $200.0(1 \mathrm{X})$ & 48.6 & 73.9 & 93.5 & 94.3 & 82.1 & 81.9 \\
\hline Untreated check & & & & - & بـ & - & 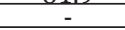 \\
\hline
\end{tabular}

$25 \%$ up to $87.5 \%$ in case of attacked area/plant (Table 2 ). For residual activity the values varied from $68.4 \%$ to $100 \%$ (Table 1 ) and from $57.8 \%$ to $99.9 \%$ (Table 2). Calculating the average values obtained from the two indicators, the greatest efficacy (99.8\% and $99 \%$,) was recorded 28 days after the treatment with spinosad (120 g a.i./ha) and emamectin-benzoate (14.25 g a.i./ ha) used in single application (Table 3). Imidacloprid efficacy, applied in a single dose (200 g a.i./ha), reached maximum 94,3\%, at 16 days after treatment, but lost the residual activity at 28 days after treatment. Insecticides applied 2 times at a lower dose gave good results also, but their efficacy increased slowly. No phytotoxic symptoms were observed. Spinosad gave similar results in some other studies performed in Brasil (Antonio Cesar dos Santos et al., 2011) and Egypt (Saad Mousa et al. 2013).

\section{CONCLUSION}

Spinosad (120 g a.i./ha) had a high activity and long lasting effect against tomato leafminer, Tuta absoluta assuring the pest control at a level comparable with emamectin-benzoate product (14.25 g a.i./ ha). Obtained results recommend this product to be introduced in the integrated tomato leafminer control on greenhouse tomatoes crops.

\section{REFERENCES}

1. Desneux N, Wajnberg E, Wyckhuys KAG, Burgio G, Arpaia S, Narváez-Vasquez C, Gonzales-Cabrera J, Catalán Ruescas D, Tabone E, Frandon J, Pizzol J, Poncet C, Cabello T, Urbaneja A (2010). Biological invasion of European tomato crops by Tuta absoluta: ecology, geographic expansion and prospects for biological control. Jour. Pest. Sci., 83:3, 197-215.

2. Santos dos A.C, Oliveira de Freitas Bueno RC, Silva Vieira S, Adeney de Freitas B (2011). Efficacy of insecticides on Tuta absoluta (Meyrick) and other pest in pole tomato. BioAssay, 6:4 (2011).

3. Saad M, Sharma A, Baiomy F, Fathi E (2013). The status of tomato leafminer; Tuta absoluta (Meyrick) (Lepidoptera, Gelenchiidae) In Egypt and potential effective pesticides. Academic J. Entomol. 6 (3): 110-115, 2013. 